EPJ Web of Conferences 60, 16008 (2013)

DOI: $10.1051 /$ epjconf $/ 20136016008$

(C) Owned by the authors, published by EDP Sciences, 2013

\title{
Intrinsic top quark properties: mass, charge and polarisation measured with the ATLAS detector at the LHC
}

\author{
Fabrice Balli ${ }^{1}$, a, on behalf of the ATLAS Collaboration \\ ${ }^{1}$ CEA-Saclay, IRFU/Service de Physique des Particules, 91191 Gif-sur-Yvette Cedex, France
}

\begin{abstract}
The top quark was discovered at the Tevatron in 1995. Measuring its intrinsic properties can provide good tests of the Standard Model. These quantities are also sensitive to models beyond the Standard Model. This document reviews the measurements, performed by the ATLAS Collaboration, of three properties of the top quark: mass, charge and polarisation. They are based on data collected with the ATLAS detector at the LHC during the year 2011 at a proton-proton centre-of-mass energy of $\sqrt{s}=7 \mathrm{TeV}$.
\end{abstract}

\section{Introduction}

The top quark is the heaviest known elementary particle. This particle plays a particular role in Particle Physics, especially in the electroweak symmetry breaking mechanism. Its properties are also very sensitive to Physics beyond the Standard Model (BSM), as numerous hypothesised particles would couple to the top quark. The measurement of the intrinsic top quark properties is therefore a powerful probe for new Physics, and the detailed studies of these parameters can possibly lead to new discoveries.

In this paper, the measurement of three properties (mass, charge and polarisation) of the top quark in $t \bar{t}$ decays with the ATLAS detector [1] are presented. The $t \bar{t}$ production at the LHC comes mainly from gluon-gluon fusion. Each top quark of the $t \bar{t}$ pair decays into a $W$ boson and a $b$-quark in almost $100 \%$ of the cases. The $W$ boson decays then either into a lepton-neutrino pair, or into a quark-antiquark pair. Depending on the decay modes of the two $W$ bosons, the $t \bar{t}$ pair decay is either semi-leptonic $(\ell+$ jets channel), fully leptonic (dilepton channel) or fully hadronic (all hadronic channel). All three measurements presented here are performed in the $\ell+$ jets channel.

Section 2 reviews the measurement of the top quark polarisation. The compatibility with the Standard Model (SM) prediction is verified. In Section 3, a measurement probing the compatibility of the observed charge with the one of a Standard Model top quark and with the one of an exotic top quark is provided. Section 4 contains details about the latest and most precise result of the top quark mass measurement performed by the ATLAS Collaboration. Finally, the summary and conclusions are given in Section 5.

a. e-mail: fabrice.balli@cern.ch

\section{Top quark polarisation}

\subsection{Introduction}

The Standard Model (SM) predicts that top quarks are produced unpolarised at the LHC because of parity conservation in QCD and the unpolarised initial state. Any deviation from this prediction is a hint of new Physics. Models beyond the SM (BSM) predicting a larger forwardbackward asymmetry than the Standard Model, as was observed at the Tevatron [2-4], should also be sensitive to this measurement. Examples of such BSM models are provided in Ref. [5] and fall into two basic classes: models with an $s$-channel vector boson and models with a $t$ channel vector boson. The top quark lifetime is approximately $0.5 \times 10^{-25} \mathrm{~s}$, implying that it does not have the time to hadronise before decaying. As a result of this, the spin information is transferred to the decay products. Thus, it is possible to measure the top quark polarisation in $\ell+$ jets events by studying the distribution of the polar angle, $\theta_{\ell}$, defined as the angle of the charged lepton coming from the leptonic $W$ boson in the rest frame of the parent top quark. This distribution is given by

$$
W\left(\cos \theta_{\ell}\right) \propto 1+\alpha_{\ell} p \cos \theta_{\ell},
$$

where $p$ is the degree of polarisation along the quantisation axis and $\alpha_{\ell}$ is the lepton spin analysing power [6] predicted to be 1 at tree level. The degree of polarisation is linked to the fraction of positively polarised top quarks $f$ by:

$$
\alpha_{\ell} p=2 f-1 \text {. }
$$

The fraction $f$ and the degree of polarisation are measured with $4.7 \mathrm{fb}^{-1}$ of data. The details of the analysis are given in Ref. [7].

\subsection{Event selection and reconstruction}

The cut-based event selection [7] is adapted to the topology of $t \bar{t}$ semi-leptonic events. It requires: 
- Exactly one isolated reconstructed lepton (electron $e$ or muon $\mu$ ) matching their respective trigger.

- At least four jets (built from electromagnetic clusters with the anti- $k_{T}$ algorithm [8] with an $R$ parameter of $0.4)$.

- Minimal values for the missing transverse momentum $\left(E_{\mathrm{T}}^{\text {miss }}\right)$ and for the leptonic $W$ candidate transverse mass ${ }^{1}$, both depending on the lepton channel.

- At least one jet is tagged as originating from a $b$-quark ( $b$-jet). The $b$-tagging is using a neural network algorithm, more details are provided in Ref. [9].

Background sources arise from various processes (single top, diboson, $W+$ jets, $Z+$ jets and multijet events with fake leptons) and are estimated from simulation, except for the normalisation of $W+$ jets contribution and multijet production with misidentified leptons, which are estimated from the data $[10,11]$.

The reconstruction of the top quark rest frame requires a full kinematic reconstruction of the event. This is performed using a kinematic likelihood fit [12] choosing the event topology that best fits the decay hypothesis. It is relying on constraints of the $W$ boson and top quark masses, the energies of the reconstructed particles and the neutrino momentum. This kinematic fit loops over all leading-jet permutations and assigns each jet to be either a $b$-jet or a light jet originating from the hadronic top quark. The $b$-tagging information is also taken into account in the likelihood function.

\subsection{Template fit}

\subsubsection{Description of the method}

Templates of the reconstructed $\cos \theta_{\ell}$ distribution are built by weighting the simulated signal events, so that the parton-level distributions are proportional to $\left(1+\cos \theta_{\ell}\right)$ and $\left(1-\cos \theta_{\ell}\right)$, corresponding to fully positively polarised and fully negatively polarised top quarks, respectively. A binned likelihood fit to the data is then performed using these templates, allowing to measure the fraction of positively polarised top quarks. Figure 1 shows the $\cos \theta_{\ell}$ distribution for the templates, for the SM prediction (corresponding to $\alpha_{\ell} p=0$ ), for the data and for the best fit to the data, in the muon channel.

\subsubsection{Systematic uncertainties}

The systematic uncertainties on $f$ are evaluated by varying the templates for each source, and repeating the fit over ensembles of pseudo-data, as described in Ref. [7]. The uncertainties arise from the reconstruction and identification of the charged lepton, the jets and the $E_{\mathrm{T}}^{\text {miss }}$, and also from the theoretical uncertainties on $t \bar{t}$ production in simulation. The dominant uncertainties come from signal modelling and jet reconstruction uncertainties.

1. Defined as $\sqrt{2 p_{\mathrm{T}, \ell} p_{\mathrm{T}, v}\left[1-\cos \left(\phi_{\ell}-\phi_{v}\right)\right]}$.

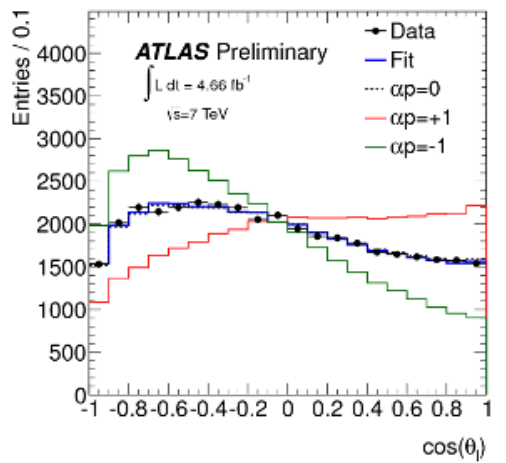

Figure 1. Result of the $\cos \theta_{\ell}$ distribution fit compared to the data in the $\mu+$ jets channel. Templates for different polarisations $\left(\alpha_{\ell} p=0, \pm 1\right)$ are also shown [7].

\subsubsection{Result}

Combining both electron and muon channels, the measured fraction of positively polarised top quarks is:

$$
f=0.470 \pm 0.009(\text { stat })_{-0.032}^{+0.023} \text { (syst) } .
$$

Within the uncertainty, this result is compatible with the SM prediction.

\section{Top quark charge}

\subsection{Introduction}

The top quark charge is predicted to be $2 e / 3$ in the Standard Model, but some exotic models [13] predict a different value, e.g. $-4 e / 3$. The semi-leptonic decay of a top quark in the SM is predicted to produce a charged lepton and a leptonic $b$-quark with opposite signs (OS), whereas they are predicted to have the same sign (SS) in the exotic scenario. The experimental difficulty is to determine the charge of $b$-quarks, as they form $b$-jets in the detector. Two methods were applied to data taken in 2011, corresponding to an integrated luminosity of $0.7 \mathrm{fb}^{-1}$. They are briefly explained in the following sections. More details on the measurement are provided in [14].

\subsection{Track charge weighting method}

The track charge weighting method is using the charges associated with those tracks which are matched to a $b$-jet. The event selection requires an additional $b$-tagged jet after the cut-based selection described in Section 2.2. Using these events with two $b$-tagged jets, the invariant mass of the each $b$-jet and the charged lepton is used to assign one $b$-jet to the leptonic $b$-quark and to perform cuts increasing the purity. The measurement is then based on the $\left\langle Q_{\text {comb }}\right\rangle=\left\langle Q_{b j e t} \cdot Q_{\ell}\right\rangle$ observable ${ }^{2}$ where $Q_{b j e t}$ is defined as follows:

$$
Q_{b j e t}=\frac{\Sigma_{i} q_{i}\left|\vec{j} \cdot \vec{p}_{i}\right|^{K}}{\Sigma_{i}\left|\vec{j} \cdot \vec{p}_{i}\right|^{\kappa}},
$$

2. $Q_{\ell}$ is the charge of the lepton. 


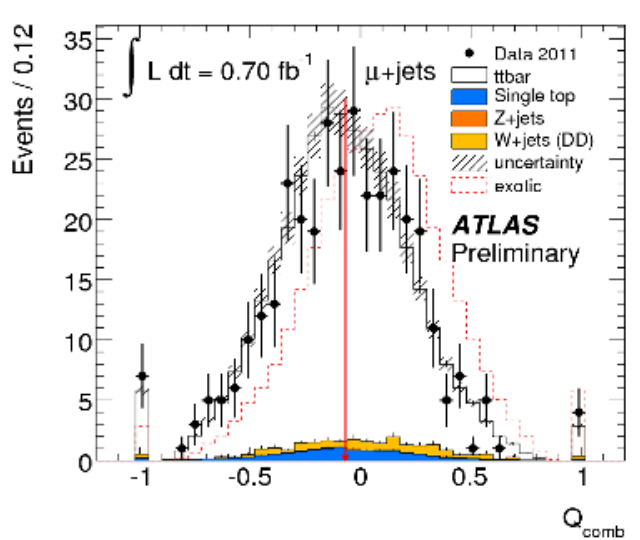

(a) $Q_{\text {comb }}$

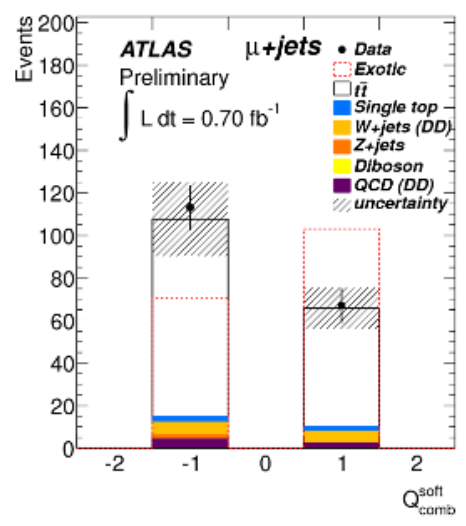

(b) $Q_{\text {comb }}^{\text {soft }}$

Figure 2. Distributions of the two observables used for the measurement in the $\mu+$ jets channel, for data, simulated SM events and simulated exotic events [14].

where $\vec{j}$ is the direction of $b$-jet axis, $q_{i}, \vec{p}_{i}$ refer to the $\mathrm{i}^{\text {th }}$ track charge and momentum, and $\kappa=0.5$. Figure 2(a) gives the distribution of this observable for the data, together with the SM and exotic model predictions in the muon channel.

\subsection{Soft muon method}

The second method uses the information of semileptonic decaying $b$-jets. The soft muon from the $b$-jet decay has the same sign as the $b$-quark it originates from. The selection of the leptonic $b$-jet relies on the kinematic fit described in Section 2.2. The $\left\langle Q_{\text {comb }}^{\text {soft }}\right\rangle=\left\langle Q_{\ell} \cdot Q_{\text {soft } t}\right\rangle$ observable $^{3}$ is used to perform the measurement. The distribution of this observable is provided in Figure 2(b) for the data, together with the SM and exotic model predictions in the muon channel.

\subsection{Results}

\subsubsection{Systematic uncertainties}

The systematic uncertainties on both observables were evaluated, including the calibration of the method, and are

\footnotetext{
3. $Q_{\text {soft } \mu}$ is the charge of the soft muon.
}

provided in Ref. [14]. They are dominated by ISR/FSR and jet energy scale uncertainties.

\subsubsection{Exclusion of a -4e/3 top quark charge}

A large number of pseudo-experiments were performed in the simulation for SM and exotic events, for both methods. More details on the statistical methods are provided in [14]. The exotic model is excluded at more than $5 \sigma$ in both methods, and the results agree with the SM prediction.

\section{Top quark mass}

\subsection{Introduction}

The top quark mass ( $\left.m_{\text {top }}\right)$ is a fundamental parameter of SM and BSM physics. It is important to measure it with the highest posssible accuracy. For example, the current precision does not allow to know whether the vacuum is in a stable or metastable state [15]. The use of this parameter in electroweak fits to test SM and BSM predictions is another example of the motivation for measuring the top quark mass precisely.

The ATLAS measurements are performed in all $t \bar{t}$ decay modes, the most precise measurement being performed in the $\ell+$ jets channel with $4.7 \mathrm{fb}^{-1}$ of data. This measurement is emphasized here, and more details about it are provided in Ref. [16]. For the measurements in the dilepton and all hadronic channels, one can refer to [17] and [18], respectively.

\subsection{The 3d-analysis}

The ATLAS measurements of $m_{\text {top }}$ in the $\ell+$ jets channel are using template methods, relying on the reconstructed hadronic top quark invariant mass $\left(m_{\mathrm{top}}^{\text {reco }}\right)$. This estimator is sensitive to the top quark mass, as seen in Figure 3(a). Unfortunately it is also sensitive to jet energy scale (JES) changes, as shown in Figure 3(b). This implies a possibly big impact of the JES uncertainty on the precision of the measurement. The second biggest potential impact on the measurement accuracy comes from the relative $b$-to-light jet energy scale (bJES), as shown in Figure 3(c). In the 3d-analysis, the JES changes are constrained with the reconstructed hadronic $W$ invariant mass $\left(m_{\mathrm{W}}^{\text {reco }}\right)$ of each event. A jet scale factor (JSF) is measured in-situ in the data, as was already done in previous ATLAS top quark mass measurements [12]. In addition, the bJES changes are constrained with the use of the $R_{\mathrm{lb}}^{\text {reco }}$ observable, defined as follows:

- $R_{\mathrm{lb}}^{\text {reco }}=\frac{p_{T}^{\text {blep }}+p_{T}^{\text {bhad }}}{p_{T}^{\text {jeet }_{1}}+p_{T}}(2 \mathrm{~b}$-tagged jet events $)$,

- $R_{\mathrm{lb}}^{\text {reco }}=\frac{p_{T}^{\text {bag }}}{\left(p_{T}^{w_{\text {jet }}}+p_{T}^{w_{\text {jet }}}\right) / 2}(1$ b-tagged jet events $)$.

This observable, being sensitive to $b$-jet energy changes (bJSF), as shown in Figure 4, allows to perform an insitu calibration of the bJES. After the selection and event reconstruction described in Section 2.2, templates of the 


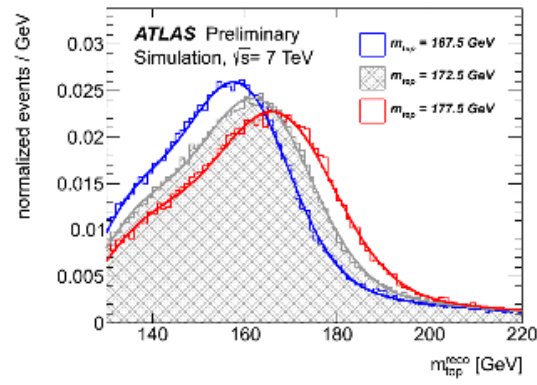

(a) $m_{\text {top }}^{\text {reco }}$ sensitivity to $m_{\text {top }}$

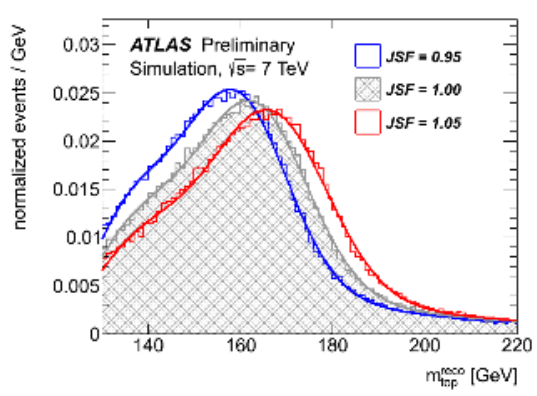

(b) $m_{\text {top }}^{\text {reco }}$ sensitivity to JSF

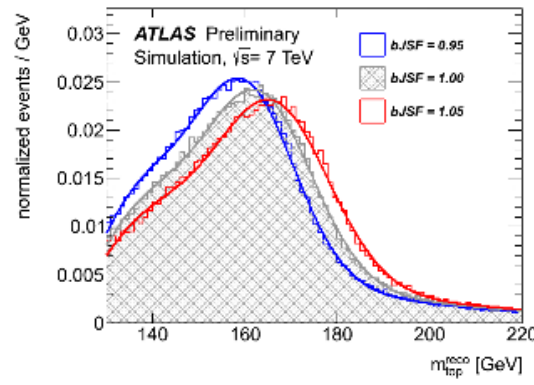

(c) $m_{\text {top }}^{\text {reco }}$ sensitivity to bJSF

Figure 3. $m_{\mathrm{top}}^{\text {reco }}$ distribution in simulated events with at least two $b$-tagged jets, for various input values of $m_{\mathrm{top}}$ (a), JSF (b) and bJSF (c). Each distribution is fitted with the sum of a Gaussian and a Landau function [16].

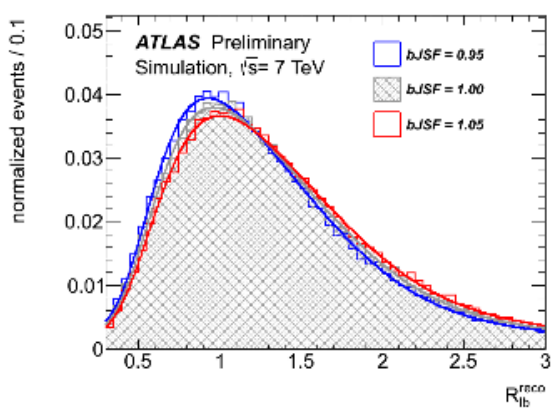

Figure 4. $R_{\mathrm{lb}}^{\text {reco }}$ distribution in simulated events with at least two $b$-tagged jets, for three different values of the input bJSF. Each distribution is fitted with the sum of a Gaussian and a Landau function [16].

three observables $\left(m_{\mathrm{top}}^{\mathrm{reco}}, m_{\mathrm{W}}^{\text {reco }}\right.$ and $\left.R_{\mathrm{lb}}^{\text {reco }}\right)$ are built for various input values of $m_{\text {top }}$, JSF and bJSF. Fitting the templates allows to obtain probability density functions for each observable as a function of the input parameters, and eventually leads to a likelihood function. An unbinned likelihood fit to the data, simultaneously for the three observables in each event, gives the measured values for $m_{\text {top }}$, JSF and bJSF. The method was verified with pseudoexperiments performed on simulated events.

\subsection{Top quark mass measurement}

\subsubsection{Systematic uncertainties}

Details on all sources of systematic uncertainty for this measurement are provided in Ref [16]. For each source of experimental uncertainty, the fit is performed on simulated events with the uncertainty source varied up and down by one standard deviation. It is repeated over 500 pseudoexperiments, and the difference with the central fitted values of the measured parameters ( $m_{\mathrm{top}}$, JSF and bJSF) in simulation is taken as the uncertainty on these parameters. The theoretical uncertainties are evaluated by fitting dedicated $t \bar{t}$ samples.

The analysis was also performed without a bJSF constraint, in order to compare with the previous ATLAS mea- surement that was using a $2 \mathrm{~d}$-analysis technique. The resulting uncertainties are given in Table 1. Comparing to the $2 \mathrm{~d}$-analysis, the major improvements in the $3 \mathrm{~d}$-analysis are seen for hadronisation, ISR/FSR and bJES uncertainties. This comes at a cost of an increase of the uncertainty due to the $b$-tagging. However, the total systematic uncertainty is reduced from 2.03 to $1.35 \mathrm{GeV}$, and the total uncertainty falls from 2.05 to $1.55 \mathrm{GeV}$. The total uncertainty is dominated by the $b$-tagging and JES uncertainties.

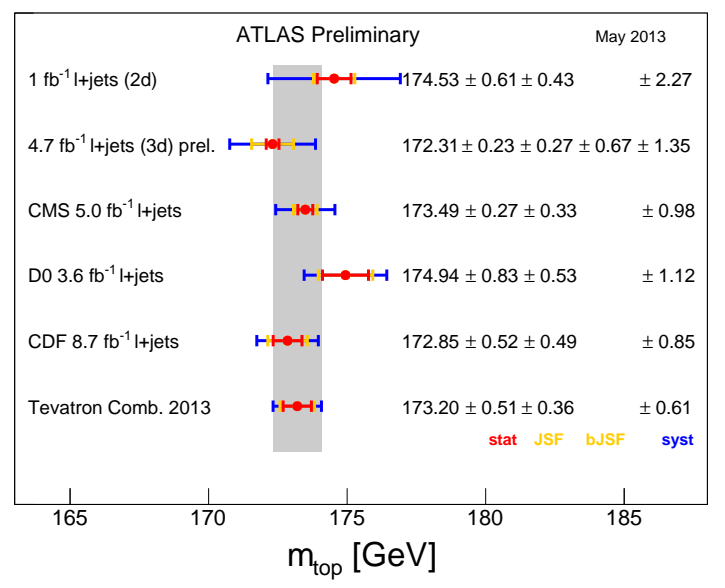

Figure 5. Result of the $3 \mathrm{~d}$-analysis for $m_{\mathrm{top}}$ compared to a number of other measurements, see Ref. [16] for details.

\subsubsection{Results}

The unbinned likelihood fit leads to the following measured values for $m_{\text {top }}$, JSF and bJSF:

$m_{\text {top }}=172.31 \pm 0.75$ (stat $\left.+\mathrm{JSF}+\mathrm{bJSF}\right) \pm 1.35$ (syst) $\mathrm{GeV}$,

$\mathrm{JSF}=1.014 \pm 0.003$ (stat) \pm 0.021 (syst),

$\mathrm{bJSF}=1.006 \pm 0.008$ (stat) \pm 0.020 (syst)

\section{Conclusion}

The ATLAS measurement of top quark polarisation leads to a value compatible with the Standard Model prediction of unpolarised top quark production at the LHC, 


\begin{tabular}{|l|r|r||r|r|r||}
\cline { 2 - 6 } \multicolumn{1}{c|}{} & \multicolumn{2}{|c||}{2 d-analysis } & \multicolumn{3}{c|}{$3 \mathrm{~d}$-analysis } \\
\cline { 2 - 6 } \multicolumn{1}{c|}{} & $m_{\text {top }}$ & JSF & $m_{\text {top }}$ & JSF & bJSF \\
\hline Measured value & 172.80 & 1.014 & 172.31 & 1.014 & 1.006 \\
\hline Data statistics & 0.23 & 0.003 & 0.23 & 0.003 & 0.008 \\
\hline Jet energy scale factor (stat. comp.) & 0.27 & $\mathrm{n} / \mathrm{a}$ & 0.27 & $\mathrm{n} / \mathrm{a}$ & $\mathrm{n} / \mathrm{a}$ \\
bJet energy scale factor (stat. comp.) & $\mathrm{n} / \mathrm{a}$ & $\mathrm{n} / \mathrm{a}$ & 0.67 & $\mathrm{n} / \mathrm{a}$ & $\mathrm{n} / \mathrm{a}$ \\
Method calibration & 0.13 & 0.002 & 0.13 & 0.002 & 0.003 \\
\hline Signal MC generator & 0.36 & 0.005 & 0.19 & 0.005 & 0.002 \\
Hadronisation & 1.30 & 0.008 & 0.27 & 0.008 & 0.013 \\
Pileup & 0.03 & 0.000 & 0.03 & 0.000 & 0.001 \\
Underlying event & 0.02 & 0.001 & 0.12 & 0.001 & 0.002 \\
Colour reconnection & 0.03 & 0.001 & 0.32 & 0.001 & 0.004 \\
ISR and FSR (signal only) & 0.96 & 0.017 & 0.45 & 0.017 & 0.006 \\
Proton PDF & 0.09 & 0.000 & 0.18 & 0.000 & 0.001 \\
\hline single top normalization & 0.00 & 0.000 & 0.00 & 0.000 & 0.000 \\
W+jets background & 0.02 & 0.000 & 0.03 & 0.000 & 0.000 \\
QCD multijet background & 0.04 & 0.000 & 0.10 & 0.000 & 0.001 \\
\hline Jet energy scale & 0.60 & 0.005 & 0.79 & 0.004 & 0.007 \\
$b$-jet energy scale & 0.92 & 0.000 & 0.08 & 0.000 & 0.002 \\
\hline$b$-tagging efficiency and mistag rate & 0.17 & 0.001 & 0.81 & 0.001 & 0.011 \\
Jet energy resolution & 0.22 & 0.006 & 0.22 & 0.006 & 0.000 \\
Jet reconstruction efficiency & 0.03 & 0.000 & 0.05 & 0.000 & 0.000 \\
Lepton energy scale & 0.03 & 0.000 & 0.04 & 0.000 & 0.000 \\
Missing transverse momentum & 0.01 & 0.000 & 0.03 & 0.000 & 0.000 \\
\hline Total systematic uncertainty & 2.03 & 0.021 & 1.35 & 0.021 & 0.020 \\
Total uncertainty & 2.05 & 0.021 & 1.55 & 0.021 & 0.022 \\
\hline
\end{tabular}

Table 1. Uncertainties on the fitted parameters for the $2 \mathrm{~d}$-analysis and $3 \mathrm{~d}$-analysis [16].

and is dominated by systematic uncertainties. The measurement of the top quark charge is also compatible with the SM expectation. The ATLAS Collaboration provides a new measurement of the top quark mass. The comparison of this measurement with the previous ATLAS and other experiments measurements is given in Figure 5.

\section{References}

[1] ATLAS Collaboration, J. Inst. 3, S08003 (2008)

[2] V.M. Abazov et al. (D0 Collaboration), Phys.Rev. D84, 112005 (2011), 1107.4995

[3] V.M. Abazov et al. (D0 Collaboration) (2012), 1207.0364

[4] T. Aaltonen et al. (CDF Collaboration), Phys.Rev. D87, 092002 (2013), 1211. 1003

[5] D. Krohn, T. Liu, J. Shelton, L.T. Wang, Phys.Rev. D84, 074034 (2011), 1105. 3743

[6] A. Brandenburg, Z. Si, P. Uwer, Phys.Lett. B539, 235 (2002), hep-ph/0205023

[7] ATLAS Collaboration, ATLAS-CONF-2012-133 (2012), http: //cds . cern. ch/record/1430034
[8] M. Cacciari, G.P. Salam, G. Soyez, JHEP 0804, 063 (2008), 0802. 1189

[9] ATLAS Collaboration, ATLAS-CONF-2011-102 (2011), http: //cds . cern. ch/record/1369219

[10] ATLAS Collaboration, Eur.Phys.J. C72, 2039 (2012), 1203.4211

[11] ATLAS Collaboration, Eur.Phys.J. C71, 1577 (2011), 1012.1792

[12] ATLAS Collaboration, Eur.Phys.J. C72, 2046 (2012), 1203. 5755

[13] D. Chang, W.F. Chang, E. Ma, Phys.Rev. D59, 091503 (1999), hep-ph/9810531

[14] ATLAS Collaboration, ATLAS-CONF-2011-141 (2011), http://cds. cern. ch/record/1385517

[15] G. Degrassi et al., JHEP 1208, 098 (2012), 1205.6497

[16] ATLAS Collaboration, ATLAS-CONF-2013-046 (2013), http: //cds . cern. ch/record/1547327

[17] ATLAS Collaboration, ATLAS-CONF-2012-082 (2012), http: //cds . cern. ch/record/1460394

[18] ATLAS Collaboration, ATLAS-CONF-2012-030 (2012), http://cds . cern. ch/record/1431895 